\title{
Diffusion MRI
}

\author{
Hidenao FUKUYAMA \\ Human Brain Research Center, Kyoto University Graduate School of Medicine \\ 54 Sogoin Kawahara-cho, Sakyo-ku, Kyoto 606-8507, Japan
}

(Received 4 December 2006 / Accepted 24 February 2007)

\begin{abstract}
Recent advances of magnetic resonance imaging have been described, especially stressed on the diffusion sequences. We have recently applied the diffusion sequence to functional brain imaging, and found the appropriate results. In addition to the neurosciences fields, diffusion weighted images have improved the accuracies of clinical diagnosis depending upon magnetic resonance images in stroke as well as inflammations.
\end{abstract}

(c) 2007 The Japan Society of Plasma Science and Nuclear Fusion Research

Keywords: diffusion, water, MRI, BOLD

DOI: $10.1585 /$ pfr.2.S1001

\section{Introduction}

MRI (magnetic resonance imaging) has great potentials to produce images containing various kinds of information from the tissue of the human body as well as animals and tissues. Recent technical advances of MRI include higher static magnetic field machine for human, and lots of MRI imaging sequences to visualize the proton and paramagnetic substances. The former enables make images of fine structures of the human brain in vivo. The latter includes susceptibility weighted image, which shows the fine magnetic susceptible conditions and with which we can see small hemorrhages in the brain parenchyma or venous dilatation. These advances have made a great impact on the imaging research as well as clinical practice. In clinical situations, T1 and T2 weighted images as well as MR angiography is the standard sequences, and T2* EPI (echo planar imaging) is utilized in functional MRI to investigate the cognitive neurosciences, which have an great success in the localizations and connections of various brain areas in the human brain.

\section{Diffusion MRI and Neurological Disorders}

Diffusion is the phenomena of random motion, or Brawnian motion of molecule. In order to visualize these phenomena by MRI, diffusion sequence was designed by Dr. Denis Le Bihan, who had been working with us for one year (6/2005-5/2006) and found the usefulness in functional brain imaging.

At present diffusion weighted images (DWI) were obtained routinely in the clinical examination for acute stroke patients to detect the very early phase of ischemia, which was reported by Gregory Sorensen, MGH, USA, in $1990 \mathrm{~s}$. This is a very sensitive image to find out the ischemic lesion as early as $30 \mathrm{~min}$ after the onset of stroke, which

author's e-mail: fukuyama@kuhp.kyoto-u.ac.jp

\section{Diffusion weighted image}

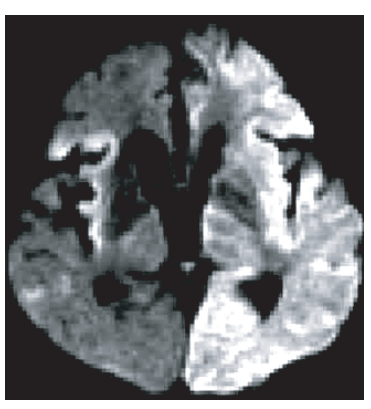

Acute Stroke

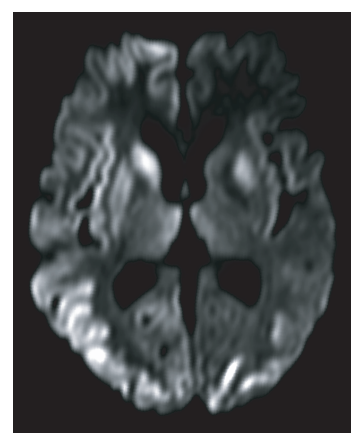

Creitzfeldt-Jacob disease
Fig. 1 Diffusion MRI in clinical cases Left side: DWI of acute stroke. Multiple embolic stroke. Right side: CreitzfeldtJacob disease. You can recognize cortical ribbon to be high intensities.

might be an important marker for cerebrovascular disorders. Ischemia of the brain occurred with a lot of etiology, and pathological changes of neuron and glia have been investigated intensively in 1980s and 1990s. These studies indicate the water accumulation in neuron and astrocyte accompanying ischemia. These changes occurred very soon after ischemic event, which makes it possible to detect the ischemic lesion with DWI [1].

DWI is able to detect abnormalities in the cerebral cortex of Creitzfeldt-Jacob disease (CJD) very specifically. CJD has been relatively difficult in diagnosis, even though EEG or clinical findings as well as gene analysis were available. DWI has a great impact on this difficult and important disorder diagnosis (Fig. 1) [2].

Therefore, DWI itself is very helpful in the clinical diagnosis of neurological disorders, especially water- 


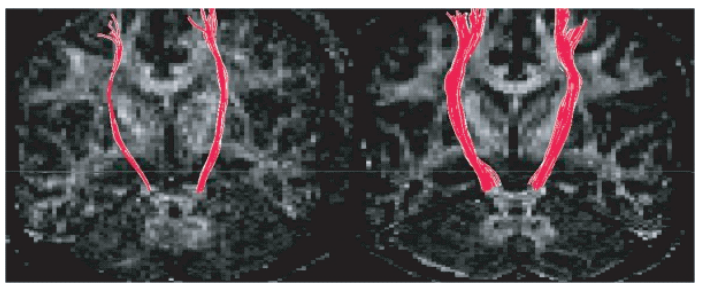

Fig. 2 Diffusion tensor imaging Left side 1.5 tesla, right side 3 tesla. Pyramidal tract was visualized using DTI studio (Dr. Mori programmed)

increased conditions such as inflammation, demyelination, ischemia, and furthermore, DWI gives us a lot of information in the brain water conditions and characters in chemical, physical, or physiological aspects. On these information, medical doctors can speculate the pathological and physiological conditions non-invasively.

\section{Fiber Tractography}

The most significant and important influence by DWI on neuroscience fields is the tracing of nerve fibers in the human brain. Previously, we have to assume the human fiber connection from the data derived from monkey or lower animal studies. This is plausible in the fact that the fine water movements occur through the nerve fibers as axonal flow. At present, we can make the tracing images socalled "tractography" by using the data obtained by DWI with more than 6 directions images, calculating the value of tensor in each voxels [3]. On these data, we can trace the fiber connections of the brain, voxel by voxel, and we can visualize such as optic radiation, pyramidal tract and so on. In case of neurosurgical resection of brain tumor or arteriovenous malformation, we can use the MRangiography as well as tractography in order to avoid sequellae of resection adjacent to the lesions (Fig. 2) [4].

\section{Diffusion fMRI}

The recent important improvement by DWI is our finding on its application to functional imaging. An fMRI depends upon the BOLD effect, which was discovered by Dr. S. Ogawa [5], that the activated area of the brain shows the increased CBF concomitant with increase of oxyhemogloboin, in contrast deoxyhemoglobin remains to be stable, therefore, paramagnetic effect of deoxyhemoglobin will decrease, following $\mathrm{T}^{*}$ image shows high signals. This image have been relatively easy to obtain using 1.5 tesla clinical use MRI machine, which makes increase on the field of research and the number of researchers. Recently, easily applicable program from UCL, statistic parametric mapping (SPM) is available freely, and then a lot of researchers are engaged in this activation studies. Easily imagined, BOLD is the phenomenon that derived from the blood supply changes of activated area, which was showed

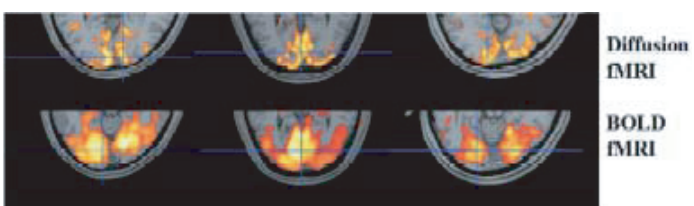

Fig. 3 Diffusion fMRI Upper raw diffusion fMRI, lower raw BOLD fMRI (conventional fMRI). Activated areas are smaller and confined to cortex in diffusion fMRI compared to BOLD fMRI.

on PET data by P. Fox, that the activated area has the small increase of oxygen consumption in comparison with the large amount of increase of CBF [6]. The critical issue of this method is not to visualize the activated neurons, but to see the blood flow changes, which was proved by Roy and Sherrington 1890. We needed to see the neuronal changes of activated neurons in some way.

In 2002, using 1.5 tesla MRI, Denis Le Bihan reported the signal changes of the visual cortex in DWI by optical stimulation [7]. DWI of 1.5 tesla is not enough to detect the water molecule movement combined with the activated neurons. The data is contaminated with noise, so they were very hard to detect the exact signals generated by the optical stimulation in the visual field.

Collaborating with Dr. Le Bihan, we could find the more accurate signal changes in the visual cortex by optical stimulation in 2005 [8]. This was done by 3 tesla machine, which has the high signal to noise ratio and we can get more stable brain response of water molecule changes after optical stimulation (Fig. 3). The precise mechanisms remain to be solved, but in vitro observation the cells of the brain tend to swell after stimulation, then we assume such kind of swelling might be derived from the water shift from free water to membrane associated water. This assumption will be able to explain our obtained result that the activated area showed the high signals in DWI. We also compared the signal change data of DWI with BOLD signal, suggesting that DWI showed the more prompt response to visual stimuli than BOLD. This suggested we assumed to see the direct changes of neurons activated, not through blood flow changes. Therefore, we assume that diffusion functional MRI will be more fruitful if we can get the good signal to noise ratio using higher static magnetic field machine.

\section{Conclusion}

Echo planar imaging technique provided us to get the MRI very quickly, just $50 \mathrm{~ms}$ for one slice in 3 tesla machine. This make possible to do experiments without radiation with regard to neuroscience and neurocognition field study. At present, new technique diffusion makes impact on neuroscience research for activation study as well as fiber connection, and also on clinical diagnosis. We expect to visualize the fiber connection or fiber conducting state 
concomitant with neuronal activation in the human brain with high tesla magnetic field MRI.

[1] G. Rordorf, W.J. Koroshetz, W.A. Copen, S.C. Cramer, P.W. Schaefer, R.F. Budzik, Jr., L.H. Schwamm, F. Buonanno, A.G. Sorensen and G. Gonzalez, Regional ischemia and ischemic injury in patients with acute middle cerebral artery stroke as defined by early diffusion-weighted and perfusion-weighted MRI. Stroke 29 (5), 939-43 (1998).

[2] Y. Shiga, K. Miyazawa, S. Sato, R. Fukushima, S. Shibuya, Y. Sato, H. Konno, K. Doh-ura, S. Mugikura, H. Tamura, S. Higano, S. Takahashi and Y. Itoyama, Diffusion-weighted MRI abnormalities as an early diagnostic marker for Creutzfeldt-Jakob disease. Neurology 63 (3), 443-9 (2004).

[3] S. Mori and J. Zhang, Principles of diffusion tensor imaging and its applications to basic neuroscience research. Neuron 51 (5), 527-39 (2006).

[4] T. Okada, N. Mikuni, Y. Miki, K. Kikuta, S. Urayama, T. Hanakawa, Y. Fushimi, A. Yamamoto, M. Kanagaki, H. Fukuyama, N. Hashimoto and K. Togashi, Corticospinal tract localization: integration of diffusion-tensor tractography at 3-T MR imaging with intraoperative white matter stimulation mapping-preliminary results. Radiology $\mathbf{2 4 0}$ (3), 849-57 (2006).

[5] S. Ogawa, T.M. Lee, A.R. Kay and D.W. Tank, Brain magnetic resonance imaging with contrast dependent on blood oxygenation. Proc Natl Acad Sci USA 87 (24), 9868-72 (1990).

[6] M.A. Mintun, B.N. Lundstrom, A.Z. Snyder, A.G. Vlassenko, G.L. Shulman and M.E. Raichle, Blood flow and oxygen delivery to human brain during functional activity: theoretical modeling and experimental data. Proc Natl Acad Sci USA 98 (12), 6859-64 (2001).

[7] D. Le Bihan, Looking into the functional architecture of the brain with diffusion MRI. Nat Rev Neurosci 4 (6), 469-80 (2003).

[8] D. Le Bihan, S. Urayama, T. Aso, T. Hanakawa and H. Fukuyama, Direct and fast detection of neuronal activation in the human brain with diffusion MRI. Proc Natl Acad Sci USA 103 (21), 8263-8 (2006). 